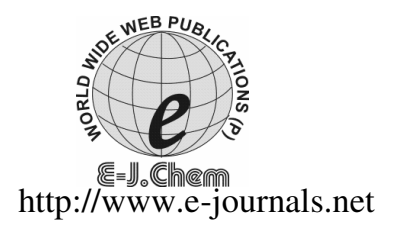

ISSN: 0973-4945; CODEN ECJHAO

E-Journal of Chemistry

2010, 7(4), 1184-1189

\title{
Ultrasonicated Synthesis of $N$-Benzyl-2,3-substituted Morpholines, via the Mitsunobu Diol Cyclisation
}

\author{
B. JAYACHANDRA REDDY and M. C. SOMASEKHARA REDDY* \\ Department of Basic Sciences, \\ G.P.R.Engg. College (Autonomous), Kurnool-518 002, (A.P), India. \\ som16@rediffmail.com
}

Received 21 September 2009; Revised 29 December 2009; Accepted 4 February 2010

\begin{abstract}
A facile five step synthesis of $N$-benzyl-2,3-substituted morpholines (i-iii) was performed. The key steps were microwave assisted Friedel-crafts acylation and diol cyclization carried out via an ultra sonication of Mitsunobu reaction using DEAD (diethylazodicarboxylate), TPP in THF for $1 \mathrm{~h}$. The morpholine products were generated as diasteriomers (ii and iii) which has been separated by the column chromatography to good yield. The structure of compounds (i-iii) has been characterized by the spectral and chemical studies.
\end{abstract}

Keywords: Friedel crafts acylation, Morpholine, Sonicator, Mitsunobu reaction, DEAD.

\section{Introduction}

Various saturated nitrogen containing heterocycles often serves as structural fragments of biological active compounds. In particular, morpholine derivatives have substance $\mathrm{P}$ antagonists (SPA), prevention of postoperative nausea and vomiting (apripitant) ${ }^{1}$, antifungal activity (amorolfin) $^{2}$, antiulcerative ${ }^{3}$ and antidepressants (viloxazine) ${ }^{4}$.

Recently, microwave ${ }^{5,}$ and ultrasonication ${ }^{6}$ assisted synthesis in organic chemistry is quickly growing. Many organic reactions proceed much faster with higher yields under microwave irradiation compared to conventional heating. It has long been know that molecules undergo excitation with electromagnetic radiation is a technique for microwave synthesis ${ }^{4}$. In the present work, at first, we aimed to examine the $\mathrm{AlCl}_{3}$ catalyzes the Friedelcrafts acylation on $\mathrm{H}$-imidazo[1,2-a]pyridine (8) efficiently under microwave irradiation in solvent free conditions.

Ultrasonication reactions enhances the reaction rates up to a million times, believed to be due small cavities (100 microns) which implode, creating tremendous heat and pressure, shock waves, and particular accelerations. In this context, we became interested to developing general and efficient synthetic methodology, a facile synthesis of morpholines (i-iii) has been carried out by the mitsunobu diol cyclization ${ }^{7}$ under ultrasonication ${ }^{6}$ Mitsunobu demonstrated that the zwitterionic adducts of triphenylphosphine (TPP) and diethyl or diisopropyl azodicarboxylate (DEAD and DIAD) activate hydroxyl groups to $\mathrm{SN}_{2}$ substitution by various nucleophiles. 


\section{Experimental}

\section{1-(2-Methylimidazo [1,2-b]pyridazin-3-yl)ethanone (3)}

A mixture of pyridazine-3-amine (1) (10 g, $105.15 \mathrm{mmol})$ and 3-chloropentane-2,4-dione (2) (21.2 g; $157.7 \mathrm{mmol})$ in ethanol $(100 \mathrm{~mL})$ was refluxed ${ }^{8}$ at $80{ }^{\circ} \mathrm{C}$ for $24 \mathrm{~h}$. The volatiles were concentrated under reduced pressure. The crude material was purified through silica gel column chromatography, eluted product with $80 \%$ ethylaceate/hexanes to afford 1-(2-methylimidazo[1,2b]pyridazin-3-yl) ethanone (3) (7.1g; 39\% yield) as a solid. Mass (e/z): $176(\mathrm{M}+1) .{ }^{\mathbf{1}} \mathbf{H}$ NMR $\left(200 \mathrm{MHz}, \mathrm{CDCl}_{3}\right): \delta 8.49-8.46(\mathrm{dd}, J=1.8,2.6 \mathrm{~Hz}, 1 \mathrm{H}), 8.00-7.95(\mathrm{dd}, J=1.6,7.6 \mathrm{~Hz}, 1 \mathrm{H}), 7.25-$ $7.18(\mathrm{~m}, 1 \mathrm{H}), 2.83(\mathrm{~s}, 3 \mathrm{H}), 2.77$ (s, 3H). Anal. Calcd. for $\mathrm{C}_{9} \mathrm{H}_{9} \mathrm{~N}_{3} \mathrm{O}: \mathrm{C}, 61.70 ; \mathrm{H}, 5.18 ; \mathrm{O}, 9.13$.

\section{2-Bromo-1-(2-methylimidazo[1,2-b]pyridazin-3-yl)ethanone (4)}

To a 1-(2-methylimidazo[1,2-b]pyridazin-3-yl)ethanone (3) $(1 \mathrm{~g}, 5.71 \mathrm{mmol})$ in a mixture of diethyl ether $(10 \mathrm{~mL})$, dichloromethane $(2 \mathrm{~mL})$ and bromine $(1.09 \mathrm{~g}, 6.85 \mathrm{mmol})$ was added drop wise at $0{ }^{\circ} \mathrm{C}$. The reaction mixture ${ }^{9}$ was warmed to RT (Room temperature) and then stirred for $2 \mathrm{~h}$. The reaction mixture was diluted with diethyl ether $(50 \mathrm{~mL})$, filtered the precipitated solids. The solids were washed with $10 \%$ ethanol/diethyl ether $(20 \mathrm{~mL})$, dried under vacuum to afford 2-bromo-1-(2-methylimidazo[1,2-b]pyridazin-3-yl)ethanone (4) (850 mg; $58 \%$ yield) as a light white solid. Mass $(e / z): 255(\mathrm{M}+1) .{ }^{1} \mathbf{H}$ NMR $\left(200 \mathrm{MHz}, \mathrm{DMSO}_{6}\right): \delta$ $8.79(\mathrm{~d}, J=3 \mathrm{~Hz}, 1 \mathrm{H}), 8.28-8.23(\mathrm{dd}, J=7.8,1.4 \mathrm{~Hz}, 1 \mathrm{H}), 7.56-7.49(\mathrm{~m}, 1 \mathrm{H}), 4.95(\mathrm{~s}, 2 \mathrm{H}), 2.64$ (s, 3H). Anal. Calcd. for $\mathrm{C}_{9} \mathrm{H}_{8} \mathrm{BrN}_{3} \mathrm{O}: \mathrm{C}, 42.54 ; \mathrm{H}, 3.17 ; \mathrm{Br}, 31.45 ; \mathrm{N}, 16.54 ; \mathrm{O}, 6.30$.

Ethyl-2-(N-benzyl-N-(2-(2-methylimidazo[1,2-b]pyridazin-3-yl)-2-oxoethyl)amino) acetate (6)

To a solution of 2-bromo-1-(2-methylimidazo[1,2-b]pyridazin-3-yl)ethanone (4) (2 g, 7.87 mmol) in acetonitrile $(20 \mathrm{~mL}), N$-benzyl glycine ethyl ester $(5)(3.7 \mathrm{~g}, 19.67 \mathrm{mmol})$ was added slowly at $0{ }^{\circ} \mathrm{C}$. The reaction mixture was warmed to RT, and then stirred for $4 \mathrm{~h}$. The volatiles were concentrated under reduced pressure; the residue was diluted with ethyl acetate and washed with water. The reaction mixture was dried (anhy. $\mathrm{Na}_{2} \mathrm{SO}_{4}$ ) concentrated under reduced pressure. The residue was purified through silica gel column chromatography, eluted product by using $20 \%$ ethyl acetate/hexanes to afford ethyl-2-( $N$-benzyl- $N$-(2-(2methylimidazo[1,2-b]pyridazin-3-yl)-2-oxoethyl)amino)acetate (6) $(2.1 \mathrm{~g}, 72.9 \%$ yield $)$ as a syrup. Mass (e/z): $367(\mathrm{M}+1) .{ }^{1} \mathbf{H}$ NMR $\left(200 \mathrm{MHz}, \mathrm{CDCl}_{3}\right): \delta 8.41-8.38(\mathrm{dd}, J=3.0,1.6$ $\mathrm{Hz}, 1 \mathrm{H}), 7.97-7.91(\mathrm{dd}, J=7.6,1.8 \mathrm{~Hz}, 1 \mathrm{H}), 7.34-7.14(\mathrm{~m}, 6 \mathrm{H}), 4.47$ (s, 2H), 4.21-4.10 (q, $2 \mathrm{H}), 4.05(\mathrm{~s}, 2 \mathrm{H}), 2.76(\mathrm{~s}, 3 \mathrm{H}), 1.25(\mathrm{t}, J=6 \mathrm{~Hz}, 3 \mathrm{H})$. Anal. Calcd. for $\mathrm{C}_{20} \mathrm{H}_{22} \mathrm{~N}_{4} \mathrm{O}_{3}: \mathrm{C}$, 65.56; H, 6.05; N, 15.29; O, 13.10.

\section{2-(N-Benzyl-N-(2-hydroxyethyl)amino)-1-(2-methylimidazo[1,2-b]pyridazin-3-yl) ethanol (7)}

To a solution of ethyl-2-( $N$-benzyl- $N$-(2-(2-methylimidazo[1,2-b]pyridazin-3-yl)-2-oxoethyl) amino) acetate $(6)(2 \mathrm{~g}, 5.46 \mathrm{mmol})$ in methanol $(15 \mathrm{~mL})$ was added $\mathrm{NaBH}_{4}(247 \mathrm{mg}, 6.55$ mmol) at $0{ }^{\circ} \mathrm{C}$, then iodine $(27 \mathrm{mg}, 0.108 \mathrm{mmol})$ was added at $0{ }^{\circ} \mathrm{C}$. The reaction mixture was warmed to RT and then stirred for $3 \mathrm{~h}$. The volatiles were concentrated under reduced pressure, then residue was diluted in ethyl acetate. The organic layer was separated, washed with water, dried (anhy. $\left.\mathrm{Na}_{2} \mathrm{SO}_{4}\right)$ and concentrated under reduced pressure to afford 2-( $N$-benzyl- $N$ - $(2-$ hydroxyethyl)amino)-1-(2-methylimidazo[1,2-b]pyridazin-3-yl)ethanol (7) (1.4 g, 79\% yield) as a syrup. Mass (e/z): $327(\mathrm{M}+1) .{ }^{1} \mathbf{H}$ NMR $\left(200 \mathrm{MHz}, \mathrm{CDCl}_{3}\right): \delta 8.18-8.15(\mathrm{dd}, J=2.8,1.6 \mathrm{~Hz}$, $1 \mathrm{H}), 7.85-7.79(\mathrm{dd}, J=7.6,1.6 \mathrm{~Hz}, 1 \mathrm{H}), 7.38-7.22(\mathrm{~m}, 5 \mathrm{H}), 6.99-6.92(\mathrm{~m}, 1 \mathrm{H}), 5.31-5.24(\mathrm{~m}$, $1 \mathrm{H}), 3.75-3.62(\mathrm{~m}, 4 \mathrm{H}), 3.30-3.19(\mathrm{~m}, 1 \mathrm{H}), 3.02-2.81(\mathrm{~m}, 3 \mathrm{H}), 2.60-2.50(\mathrm{~m}, 1 \mathrm{H}), 2.42(\mathrm{~s}, 3 \mathrm{H})$. Anal. Calcd for $\mathrm{C}_{18} \mathrm{H}_{22} \mathrm{~N}_{4} \mathrm{O}_{2}$ : C, 66.24; H, 6.79; N, 17.17; O, 9.80. 


\section{3-(4-Benzylmorpholin-2-yl)-2-methylimidazo[1,2-b]pyridazine (i)}

2-(N-Benzyl- $N$-(2-hydroxyethyl)amino)-1-(2-methylimidazo[1,2-b]pyridazin-3-yl)ethanol (7) (250 mg, $1.106 \mathrm{mmol})$ and TPP (376 mg, $1.437 \mathrm{mmol})$ in anhy.THF (5 mL) was ultra sonicated for $15 \mathrm{~min}$, then DEAD (288 $\mathrm{mg}, 1.659 \mathrm{mmol})$ was added drop wise to the reaction mixture over the $5 \mathrm{~min}$. After addition, reaction mixture was ultra sonicated for $45 \mathrm{~min}$ at RT. The volatiles were concentrated under reduced pressure, then the residue was purified through silica gel column chromatography, eluted product with $70 \%$ ethyl acetate/hexane afford 3-(4-benzylmorpholin-2-yl)-2-methylimidazo[1,2-b]pyridazine (i) (134 mg, 57\% yield) as syrup. Mass (e/z): $309(\mathrm{M}+1) .{ }^{1} \mathbf{H}$ NMR $\left.\left(200 \mathrm{MHz}, \mathrm{CDCl}_{3}\right):\right): \delta$ 8.29-8.26 (dd, $J=2.8,1.8 \mathrm{~Hz}, 1 \mathrm{H}), 7.83-7.81(\mathrm{dd}, J=7.2,1.8 \mathrm{~Hz}, 1 \mathrm{H}), 7.38-7.22(\mathrm{~m}, 5 \mathrm{H})$, 6.99-6.92 (m, $1 \mathrm{H}), 5.37-5.30(\mathrm{dd}, J=7.6,2.8 \mathrm{~Hz}, 1 \mathrm{H}), 4.07-3.81(\mathrm{~m}, 2 \mathrm{H}) ,3.62(\mathrm{~s}, 3 \mathrm{H})$, 2.92-2.74 (m, 3H), $2.59(\mathrm{~s}, 3 \mathrm{H}), 2.45-2.32(\mathrm{~m}, 1 \mathrm{H})$. Anal. Calcd for $\mathrm{C}_{18} \mathrm{H}_{20} \mathrm{~N}_{4} \mathrm{O}: \mathrm{C}, 70.11$; $\mathrm{H}, 6.54 ; \mathrm{N}, 18.17 ; \mathrm{O}, 5.19$.

\section{1-(H-Imidazo[1,2-a]pyridin-3-yl)propan-1-one (10)}

A mixture of $H$-imidazo[1,2-a]pyridine $(\mathbf{8})(500 \mathrm{mg}, 4.23 \mathrm{mmol})$, propanoic anhydride (9) $(2.75 \mathrm{~g}, 21.18 \mathrm{mmol})$ and $\mathrm{AlCl}_{3}(1.12 \mathrm{~g}, 8.46 \mathrm{mmol})$ was irradiated in microwave ${ }^{10}$ for 20 min at $100{ }^{\circ} \mathrm{C}$. The crude was neutralized with sat. $\mathrm{Na}_{2} \mathrm{CO}_{3}$ and extracted product into ethyl acetate $\left(25 \mathrm{~mL}\right.$ ), dried (anhy. $\mathrm{Na}_{2} \mathrm{SO}_{4}$ ) and concentrated under reduced pressure. The crude material was purified through silica gel column chromatography, eluted product with $2 \%$ $\mathrm{MeOH} / \mathrm{DCM}$ to afford 1-(H-imidazo[1,2-a]pyridin-3-yl)propan-1-one (10) (352 mg, 47.7\% yield) as a solid. Mass $(e / z): 175(\mathrm{M}+1) .{ }^{1} \mathbf{H}$ NMR $\left(200 \mathrm{MHz}, \mathrm{DMSO}_{\mathrm{d}}\right): \delta 8.56(\mathrm{~d}, J=6.6$ $\mathrm{Hz}, 1 \mathrm{H}), 8.63(\mathrm{~s}, 1 \mathrm{H}), 7.84(\mathrm{~d}, J=9.2 \mathrm{~Hz}, 1 \mathrm{H}), 7.63(\mathrm{t}, J=6.8 \mathrm{~Hz}, 1 \mathrm{H}), 7.26(\mathrm{t}, J=6.6$ $\mathrm{Hz}, 1 \mathrm{H}), 3.04-2.93(\mathrm{q}, 2 \mathrm{H}), 1.15(\mathrm{t}, J=7.4 \mathrm{~Hz}, 3 \mathrm{H})$. Anal. Calcd for $\mathrm{C}_{10} \mathrm{H}_{10} \mathrm{~N} 2 \mathrm{O}: \mathrm{C}$, 68.95; H, 5.79; N, 16.08; O, 9.18.

\section{2-Bromo-1-(H-imidazo[1,2-a]pyridin-3-yl)propan-1-one (11)}

Compound (11) was prepared according to the method describes for preparation of compound (4), Pale brown Solid. Mass (e/z): $255(\mathrm{M}+1) .{ }^{1} \mathbf{H}$ NMR $\left(200 \mathrm{MHz}, \mathrm{CDCl}_{3}\right): \delta$ $9.82(\mathrm{~d}, J=6.6 \mathrm{~Hz}, 1 \mathrm{H}), 9.24(\mathrm{~s}, 1 \mathrm{H}), 8.60(\mathrm{~d}, J=9.2 \mathrm{~Hz}, 1 \mathrm{H}), 8.15(\mathrm{t}, J=6.8 \mathrm{~Hz}, 1 \mathrm{H})$, $7.62(\mathrm{t}, J=6.6 \mathrm{~Hz}, 1 \mathrm{H}), 5.51-5.59(\mathrm{q}, 2 \mathrm{H}), 2.01(\mathrm{~d}, J=7.4 \mathrm{~Hz}, 3 \mathrm{H})$. Anal. Calcd. for $\mathrm{C}_{10} \mathrm{H}_{9} \mathrm{BrN}_{2} \mathrm{O}: \mathrm{C}, 47.46 ; \mathrm{H}, 5.79 ; \mathrm{N}, 11.07 ; \mathrm{O}, 6.32$.

Ethyl 2-(N-(1-H-imidazo[1,2-a]pyridin-3-yl)-1-oxopropan-2-yl)-N-benzylamino) acetate (13)

Compound (13) was prepared according to the method describes for preparation of compound (6), Syrup. Mass (e/z): $255(\mathrm{M}+1) .{ }^{1} \mathbf{H}$ NMR $(200 \mathrm{MHz}, \mathrm{CDCl} 3): \delta 9.68(\mathrm{~d}, \mathrm{~J}=7$ $\mathrm{Hz}, 1 \mathrm{H}), 8.56(\mathrm{~s}, 1 \mathrm{H}), 7.76(\mathrm{~d}, J=9 \mathrm{~Hz}, 1 \mathrm{H}), 7.46(\mathrm{t}, J=7.8 \mathrm{~Hz}, 1 \mathrm{H}), 7.34-7.26(\mathrm{~m}, 5 \mathrm{H})$, $7.11(\mathrm{t}, J=5.6 \mathrm{~Hz}, 1 \mathrm{H}), 4.21-4.07(\mathrm{q}, 2 \mathrm{H}), 3.84(\mathrm{~d}, J=5.6 \mathrm{~Hz}, 1 \mathrm{H}), 3.81(\mathrm{~s}, 3 \mathrm{H}), 3.41(\mathrm{~s}$, $2 \mathrm{H}), 1.40(\mathrm{~d}, J=6.8 \mathrm{~Hz}, 3 \mathrm{H}), 1.27(\mathrm{t}, J=7 \mathrm{~Hz}, 3 \mathrm{H})$. Anal. Calcd for $\mathrm{C}_{21} \mathrm{H}_{23} \mathrm{~N}_{3} \mathrm{O}_{3}: \mathrm{C}, 69.02$; $\mathrm{H}, 6.34 ; \mathrm{N}, 11.50 ; \mathrm{O}, 13.13$.

\section{2-(N-Benzyl-N-(2-hydroxyethyl)amino)-1-(H-imidazo[1,2-a]propan-1-ol (14)}

Compound (14) was prepared according to the method describes for preparation of compound (7), Syrup. Mass $(e / z): 326(\mathrm{M}+1) .{ }^{1} \mathbf{H}$ NMR $(200 \mathrm{MHz}, \mathrm{CDCl} 3): \delta 7.85(\mathrm{~d}, \mathrm{~J}=$ $6.8 \mathrm{~Hz}, 1 \mathrm{H}), 7.52-7.11(\mathrm{~m}, 7 \mathrm{H}), 7.18(\mathrm{t}, J=6.8 \mathrm{~Hz}, 1 \mathrm{H}), 6.61(\mathrm{t}, J=6.8 \mathrm{~Hz}, 1 \mathrm{H}), 5.30(\mathrm{br}$ $\mathrm{s}, 1 \mathrm{H}), 4.86(\mathrm{t}, J=5 \mathrm{~Hz}, 1 \mathrm{H}), 4.78(\mathrm{~d}, J=9.8 \mathrm{~Hz}, 1 \mathrm{H}), 3.97(\mathrm{~d}, J=13.4 \mathrm{~Hz}, 1 \mathrm{H}), 3.69-3.44$ $(\mathrm{m}, 3 \mathrm{H}), 3.17-3.09(\mathrm{~m}, 1 \mathrm{H}), 2.86-2.72(\mathrm{~m}, 1 \mathrm{H}), 2.55-2.42(\mathrm{~m}, 1 \mathrm{H}), 0.77(\mathrm{~d}, J=6.6 \mathrm{~Hz}, 3 \mathrm{H})$. Anal. Calcd for $\mathrm{C}_{19} \mathrm{H}_{23} \mathrm{~N}_{3} \mathrm{O}_{2}: \mathrm{C}, 70.13 ; \mathrm{H}, 7.12 ; \mathrm{N}, 12.91 ; \mathrm{O}, 9.83$. 


\section{3-(4-Benzyl-3-methylmorpholin-2yl)H-imidazo[1,2-a]pyridine (ii and iii)}

2-( $N$-benzyl- $N$-(2-hydroxyethyl)amino)-1-( $H$-imidazo[1,2-a]pyridin-3-yl)propan-1-ol (14) (500 mg, $1.53 \mathrm{mmol})$ and TPP $(524 \mathrm{mg}, 1.99 \mathrm{mmol})$ in anhy.THF $(10 \mathrm{~mL})$ was ultra sonicated for $15 \mathrm{~min}$, then $\operatorname{DEAD}^{6}(401 \mathrm{mg}, 2.30 \mathrm{mmol}$ ) was added drop wise to the reaction mixture over the $5 \mathrm{~min}$. After addition, reaction mixture was ultra sonicated for $45 \mathrm{~min}$ at RT. The volatiles were concentrated under reduced pressure, then the residue was purified through silica gel column chromatography, eluted faster product with $40 \%$ ethyl acetate/hexane afford 3cis( \pm )-4-benzyl-3-methylmorpholin-2yl) $H$-imidazo[1,2-a]pyridine (ii) $(130 \mathrm{mg}, 27.5 \%$ yield) and slower product with 50\% ethyl acetate/hexanes to afford 3-trans( \pm )-4-benzyl-3methylmorpholin-2yl) $\mathrm{H}$-imidazo[1,2-a]pyridine (iii) (160 mg, 33.8\% yield ) as a syrup.

3-cis( \pm )-4-Benzyl-3-methylmorpholin-2yl)H-imidazo[1,2-a]pyridine (ii)

Mass (e/z): $308(\mathrm{M}+1)$. added ${ }^{1} \mathrm{H}$ NMR (200 MHz, DMSO-d $\left.{ }_{6}\right): \delta 8.39(\mathrm{~d}, J=6.8 \mathrm{~Hz}, 1 \mathrm{H}), 7.73(\mathrm{~s}$, $1 \mathrm{H}), 7.57(\mathrm{~d}, J=9 \mathrm{~Hz}, 1 \mathrm{H}), 7.44-7.212(\mathrm{~m}, 6 \mathrm{H}), 6.93(\mathrm{t}, J=6.8 \mathrm{~Hz}, 1 \mathrm{H}), 5.21(\mathrm{~d}, J=3 \mathrm{~Hz}, 1 \mathrm{H}), 3.88$ $(\mathrm{d}, J=13.6 \mathrm{~Hz}, 1 \mathrm{H}), 3.71-3.45(\mathrm{~m}, 2 \mathrm{H}), 3.39(\mathrm{~d}, J=13.6 \mathrm{~Hz}, 1 \mathrm{H}), 3.12-3.09(\mathrm{~m}, 1 \mathrm{H}), 2.67-2.59(\mathrm{~m}, 1$ $\mathrm{H}), 1.06(\mathrm{~d}, J=6.6 \mathrm{~Hz}, 3 \mathrm{H})$. Anal. Calcd for $\mathrm{C}_{19} \mathrm{H}_{21} \mathrm{~N}_{3} \mathrm{O}: \mathrm{C}, 74.24 ; \mathrm{H}, 6.89 ; \mathrm{N}, 13.67 ; \mathrm{O}, 5.20$.

\section{3-trans( \pm )-4-Benzyl-3-methylmorpholin-2yl)H-imidazo[1,2-a]pyridine (iii)}

Mass (e/z): $308(\mathrm{M}+1) .{ }^{1} \mathbf{H}$ NMR $\left(200 \mathrm{MHz}, \mathrm{DMSO}-\mathrm{d}_{6}\right): \delta 8.51(\mathrm{~d}, J=6.8 \mathrm{~Hz}, 1 \mathrm{H}), 7.61(\mathrm{~s}$, $1 \mathrm{H}), 7.57(\mathrm{~d}, J=9 \mathrm{~Hz}, 1 \mathrm{H}), 7.37-7.21(\mathrm{~m}, 6 \mathrm{H}), 6.93(\mathrm{t}, J=6.8 \mathrm{~Hz}, 1 \mathrm{H}), 4.73(\mathrm{~d}, J=8.2 \mathrm{~Hz}$, $1 \mathrm{H}), 4.08(\mathrm{~d}, J=13.4 \mathrm{~Hz}, 1 \mathrm{H}), 3.71-3.66(\mathrm{~m}, 2 \mathrm{H}), 3.27(\mathrm{~d}, J=13.4 \mathrm{~Hz}, 1 \mathrm{H}), 3.00-2.92(\mathrm{~m}$, $1 \mathrm{H}), 2.65-2.59(\mathrm{~m}, 1 \mathrm{H}), 2.40-2.27(\mathrm{~m}, 1 \mathrm{H}), 1.08(\mathrm{~d}, J=6.6 \mathrm{~Hz}, 3 \mathrm{H})$. Anal. Calcd for $\mathrm{C}_{19} \mathrm{H}_{21} \mathrm{~N}_{3} \mathrm{O}: \mathrm{C}, 74.24 ; \mathrm{H}, 6.89 ; \mathrm{N}, 13.67 ; \mathrm{O}, 5.20$.

\section{Results and Discussion}

All the three new compounds (i-iii) were synthesized. Cyclic keto compound (3) was prepared by cyclization of 2-amino pyridine (1) with 3-chloropentane-2,4-dione (2) in ethanol under the reflux for $24 \mathrm{~h}$ (ca. 39\% yield). Bromination of (3) in ether/dichloromethane for $2 \mathrm{~h}$ at RT to give $\alpha$-bomo compound (4) (ca. 58\% yield), then $N$-alkylation ${ }^{11}$ on $N$-benzyl glycine ester by using excess amine (5) in THF at reflux for $12 \mathrm{~h}$, in (ca. 70\% yield). Sodium borohydride mediated reduction using $I_{2}$ catalytic $^{12,13}$ yielded diole (7) in (ca. $65 \%$ yield). Then ultrasonicated mitsunobu diole cyclization ${ }^{6,7}$ to give the morpholine derivative (i) using DEAD, TPP, in THF (cat. 57\% yield) according to Scheme 1 .<smiles>CC(=O)C(Cl)C(C)=O</smiles>

2

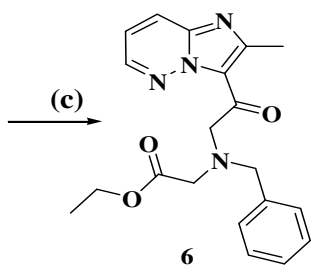

(a)<smiles>CC(=O)c1c(C)nc2cccnn12</smiles>

(d)

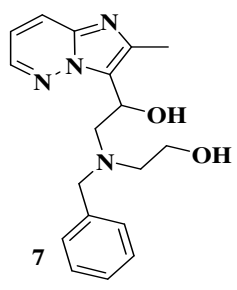<smiles>CCOC(=O)CNCc1ccc([As]c2nc3cccnn3c2C(=O)CBr)cc1</smiles>

(e)

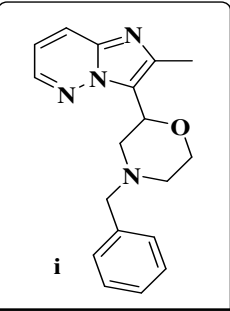

Reagents \& Conditions: (a) EtOH; rfx; 24 h (b) $\mathrm{Br}_{2}$; ether; DCM; $0^{\circ} \mathrm{C}-\mathrm{RT} ; 2$ h (c) ACN; $0{ }^{\circ} \mathrm{C}$ RT; 2 h (d) $\mathrm{NaBH}_{4} ; \mathrm{I}_{2}$; MeOH; RT; 3 h (e) DEAD; TPP; THF; Ultra sonicator; RT, 1 h

\section{Scheme 1}


A microwave synthesis ${ }^{10}$ of Friedel-crafts acylation ${ }^{5,14}$ on $H$-imidazo[1,2-a]pyridine (8) by using $\mathrm{AlCl}_{3}$, propanoic anhydride (9) irradiating in microwave at $100{ }^{\circ} \mathrm{C}$ for $20 \mathrm{~min}$ to give acylated product (10) (in ca. 45\% yield). Then, further 11, 13, 14 and morpholine derivatives (ii-iii) were prepared as followed similar procedure of related analogue 4, 6, 7 and (i). The morpholine derivatives cis (ii) and trans (iii) separated by column chromatography to afford in good yield according to Scheme 2.

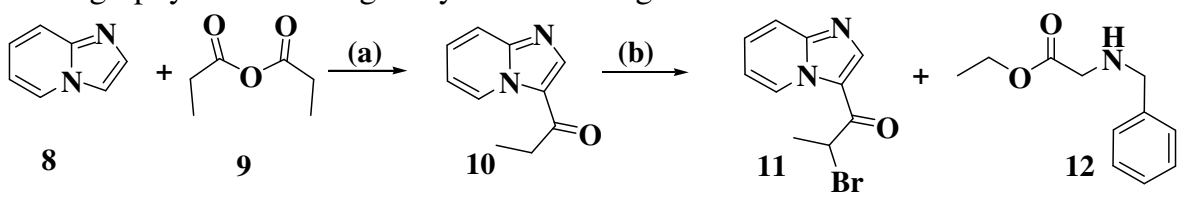

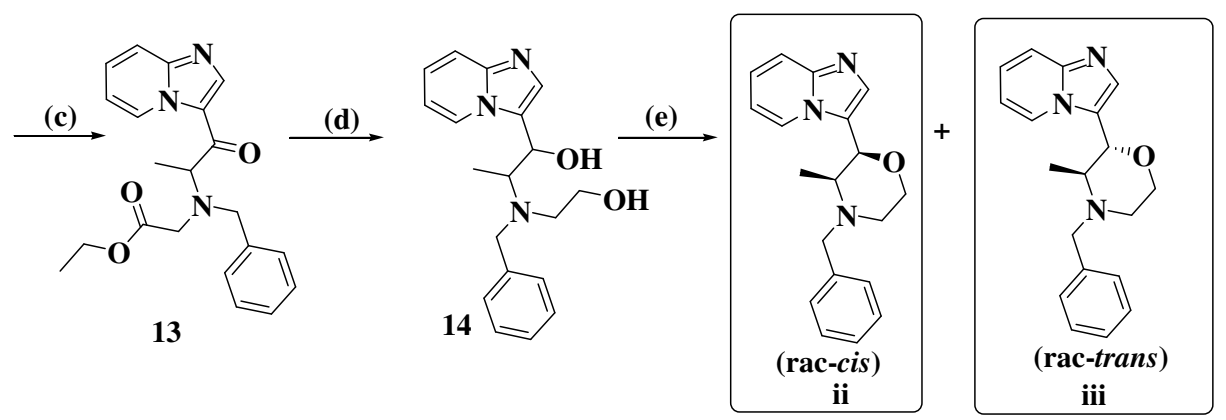

Reagents \& Conditions: (a) $\mathrm{AlCl}_{3}$; microwave; $100{ }^{\circ} \mathrm{C} ; 20 \mathrm{~min}$ (b) $\mathrm{Br}_{2}$; ether; DCM; $0^{\circ} \mathrm{C}-\mathrm{RT} ; 2 \mathrm{~h}$ (c) ACN; $0{ }^{\circ} \mathrm{C}-\mathrm{RT} ; 2 \mathrm{~h}$ (d) $\mathrm{NaBH}_{4} ; \mathrm{I}_{2} ; \mathrm{MeOH} ; \mathrm{RT} ; 3 \mathrm{~h}$ (e) DEAD; TPP; THF; Ultra sonicator; $1 \mathrm{~h}$

\section{Conclusion}

\section{Scheme 2}

The Mitsunobu diol cyclization in ultrasonicator has been given in good yields (cal. 57\%) comparatively the standard reaction conditions, where reaction proceeded in less time with good yield. When the reaction carried out at standard reaction conditions using same reagents, stirring in maganetic stirrer for $12 \mathrm{~h}$, also not reached good yield (cal. $20 \%$ ) as comparative with the same reaction in ultrasonicator for $1 \mathrm{~h}$. However, due to the explosive hazards of azodicarboxylates, we are not recommend that the sonication procedure for bigger scale $(>2 \mathrm{~g})$ unless precautions are taken to remove excessive heat buildup during the reaction.

The NMR analysis of ultrasonicated Mitsunobu diol cyclization showing diasteriomeric mixture (ii and iii) suggests that it consist almost 1:1 ratio of cis/trans. In the NMR spectra $^{15,16}$ of cis (ii), the chemical shift of the $\mathrm{C}_{2}-\mathbf{H}$ falls in the expected range $\left(\mathrm{C}_{2}-\mathbf{H}\right.$ at 5.21). The NMR of trans (iii) showed that the chemical shift of the $C_{2}-\mathbf{H}$ was shifted up field to $4.73 \mathrm{ppm}$. These shifts are consistent with the indicated structural assignments. When the proton in cis exposed to shielding effect to heterocyclic ring. The coupling of $\mathrm{C}_{2^{-}}$$\mathbf{H}$ with neighboring proton and splitting as doublet, coupling constant $(J)^{17,18}$ of cis (ii) $3 \mathrm{~Hz}$ and trans (iii) $8.2 \mathrm{~Hz}$. Comparatively of both coupling constants expected cis (ii) showing less coupling constant than corresponding trans (iii).

\section{Acknowledgment}

The authors wish to thank Principal, Director and Management of G.P.R. Engg. College 
(Autonomous), Kurnool-518 002 (A.P.), India for their constant encouragement and help. The authors are also thankful to Indian Institute of Chemical Technology (IICT), Hyderabad for spectral analysis and Literature assistance.

\section{References}

1. Keller M, Montgomery S, Ball W, Morrison M, Snavely D, Liu G, Hargreaves R, Hietala J, Lines C and Beebe K, Biological Psychiatry, 2006, 59(3), 216-223.

2. Nishiyama Y, Asagi Y, Hirani T, Yamaguchi H, Yamada N and Osumi M, Cinical and Experimental Dermatology, 2006, 17, s1, 13-17.

3. Annamaria Molnar, Ferenc Faigl, Benzamin Podanyi, Zoltan Finta, Laszlo Balazs and Istvan Hermecz, Heterocycles, 2009, 78, 10.

4. Pisani F, Fazio A, Artesi C, Russo M, Trio R, Oteri G, Perucca E and Di perri, $J$ Neurol Neutosurg Psychiatry, 1992, 55(2), 126-127.

5. Wei Deng, Yu Xu and Qing Xiang Guo, Chinese Chem lett., 2005, 16(3), 327-330.

6. Lepore S D, He Y, J Org Chem., 2003, 68, 8261-8263.

7. Wilkinson, Mark C, Bell, Rebecca, Rober, Nikiforov, Petar O, Walker and Andrew J, Synlett., 2006, 13, 2151-2153.

8. Masahiko Hayakawa, Hiroyuki Kaizawa, Ken-ichi Kawaguchi, Norko Ishikawa, Tomonobu Koizumi, Takahide Ohishi, Mayumi Yamano, Minoru Okada, Mitsuaki Ohta, Shin-ichi Tsukamoto, Florence I, Raynaud, Michael D, Waterfiledm, Peter Parker and Paul Workman, Bioorg Med Chem., 2007, 15(1), 403-412.

9. Taeyoung Choi and Eunsook Ma, Molecules, 2007, 12, 74-85.

10. Olayinka O, Ajani, Craig A, Obafemi, Chinwe O, lkpo, Kolawole O, Ajanaku, Kehinde O, Ogunniran and Oladele Oludola James, Int J Phys Sci., 2009, 4(4), 156-164.

11. Maria Sol Shmidt, Ana Maria Reverdito, Lautaro Kremenchuzky, Isabel Amalia Perillo and Maria Mercedes Blanco, Molecules, 2008, 13, 831-840.

12. Bhanu Prasad A S, Bhaskar Kanth J V and Mariappan Periasamy, Tetrahedron, 1992, 48(22), 4623-4628.

13. De Souza and Dodd M. V N, Heterocycles, 1998, 47, 811.

14. Bensari A and Zaveri N T, Synthesis, 2003, 267-271.

15. Thomas W, von Geldern, Andrew S, Tasker, Bryan K, Sorensen, Martin Winn, Bruce G, Szczepankiewicz, Douglas B, Dixon, William J, Chiou, Liming Wang, Jerry L, Wessale, Andy Adler, Kennan C, Marsh, Bach Nguyen and Terry J Opgenorth, $J$ Med Chem., 1999, 42(18), 3668-3678.

16. Gang Liu, Natasha S, Kozmina, Martina Winn, Thomas W, von Geldern, William J, Chiou, Douglas B, Dixon, Bach Nguyen, Kennan C, Marsh, Terry J and Opgenorth, J Med Chem., 1999, 42, 3679-3689.

17. Frost D J and Gunstone F D, Chem Phys Lipids, 1975, 15, 53-85.

18. Gunstone F D and Ismail IA, Chem Phys Lipids, 1967, 1, 337-340. 


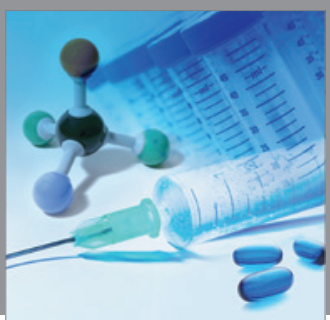

International Journal of

Medicinal Chemistry

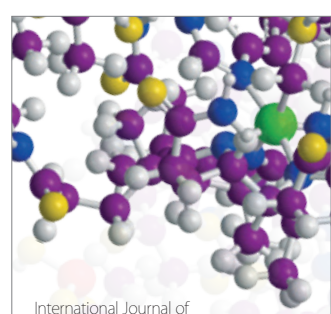

Carbohydrate Chemistry

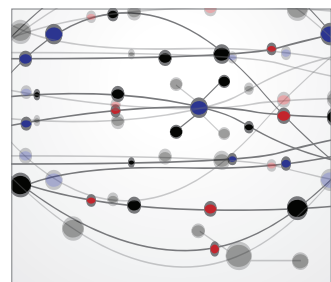

The Scientific World Journal
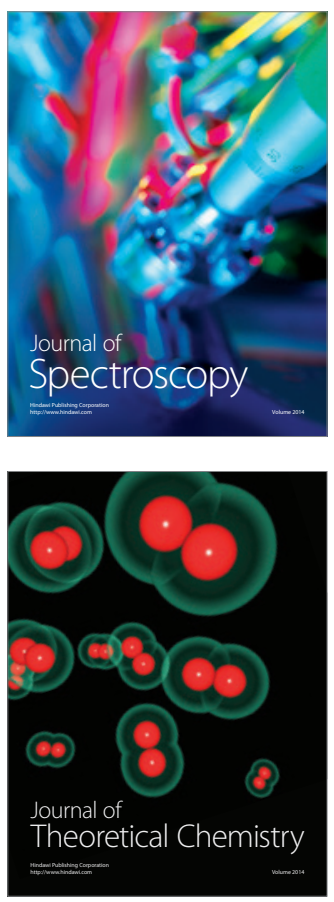
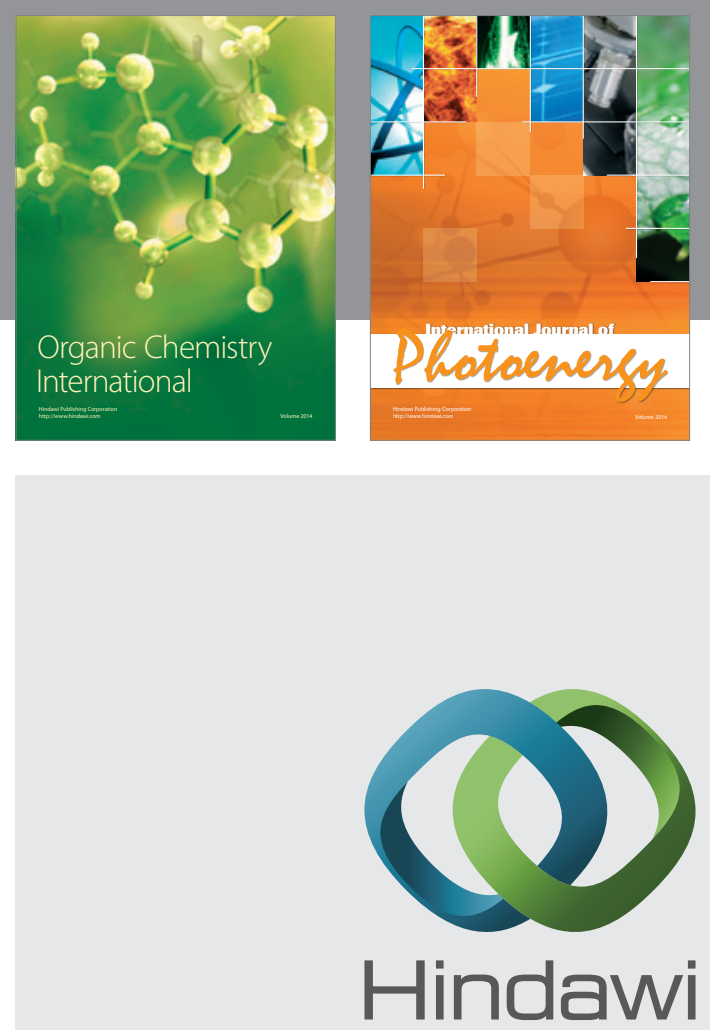

Submit your manuscripts at

http://www.hindawi.com
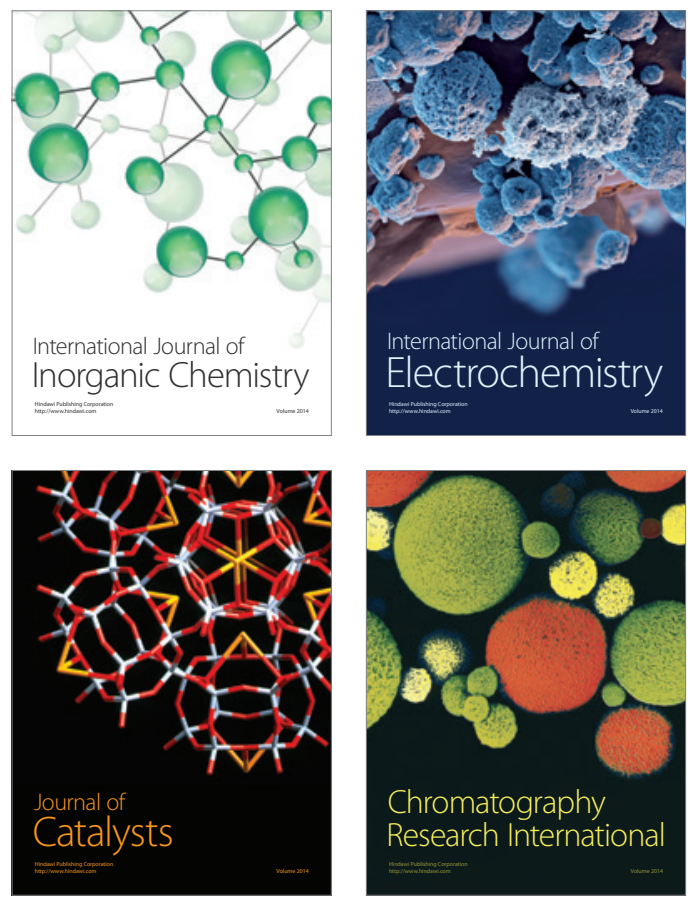
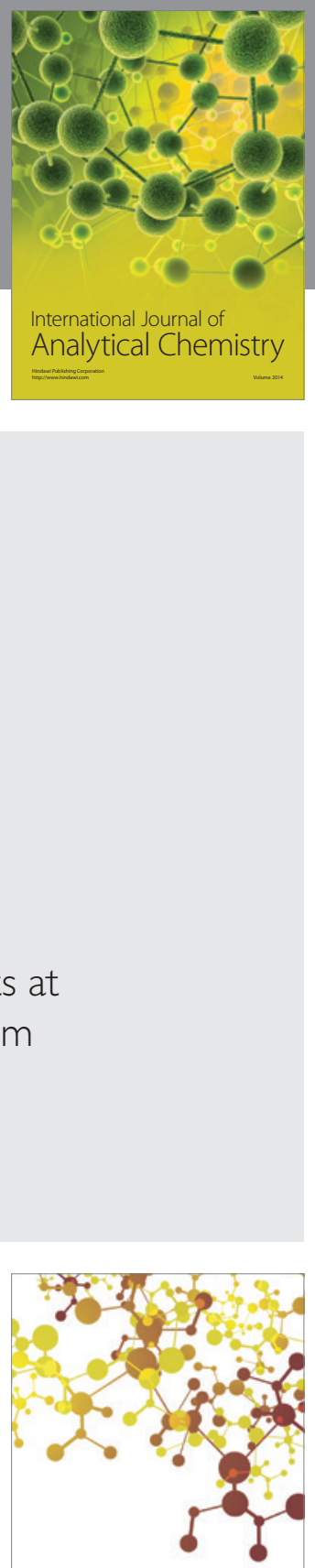

Journal of

Applied Chemistry
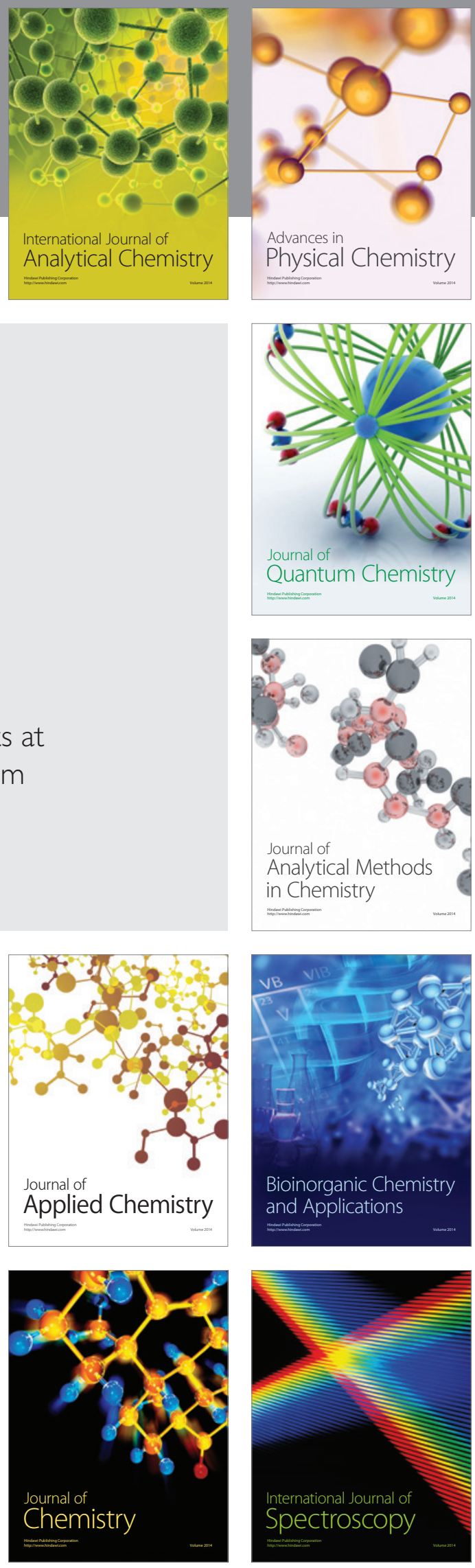